\title{
Which one is safer - performing a laparoscopic hysterectomy with a tissue fusion device involving diagnostic cystoscopy or traditional abdominal hysterectomy with ureteral dissection?
}

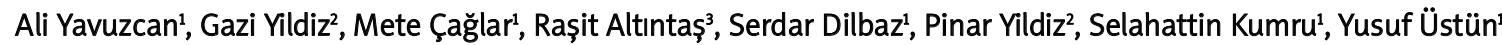 \\ 1Department of Obstetrics and Gynecology, Düzce University, Düzce, Turkey \\ 2Department of Obstetrics and Gynecology, Bucak State Hospital, Burdur, Turkey \\ ${ }^{3}$ Department of Urology, Bucak State Hospital, Burdur, Turkey
}

Videosurgery Miniinv 2013; 8 (4): 280-288

DOI: 10.5114/wiitm.2013.39504

\begin{abstract}
Introduction: It still remains an unanswered question whether, in the absence of gynecological malignancy and under elective conditions, to perform abdominal hysterectomy (AH), offering a safer approach, or to perform a laparoscopic hysterectomy $(\mathrm{LH})$ procedure.

Aim: We aimed to compare LH operations performed with a tissue fusion device accompanied by intraoperative diagnostic cystoscopy with traditional AH operations involving bilateral ureteral dissection.

Material and methods: The integrity of the ureters, ureteral peristalsis and the diameter of the ureters were examined during $\mathrm{AH}$ by inspection. At the end of $\mathrm{LH}$, the bladder wall was systematically evaluated by cystoscopy and a jet of urine spurting was noted from both ureteral orifices.

Results: The operation time was longer in patients who underwent $L H+C Y S(p=0.0001)$. The decline in hematocrit and hemoglobin levels in the postoperative period was significantly higher in patients who underwent $A H+U D(p=$ 0.0001 and $p=0.002$, respectively). No significant difference was found between the two groups in terms of ureteral injury, bowel injury or bladder injury ( $p=0.378, p=1.000$ and $p=1.000$, respectively). There was no statistically significant difference between the two groups in terms of mean body temperature and postoperative blood transfusion requirements ( $p=0.051$ and $p=0.210$, respectively). Mean parenteral analgesic requirement and length of hospital stay were significantly different between the groups ( $p=0.005$ and $p=0.0001)$. No statistically significant difference was found between the two groups in terms of postoperative cardiopulmonary complications, re-operation rate or occurrence of genitourinary fistula ( $p=1.000, p=1.000$ and $p=1.000$, respectively).

Conclusions: We concluded that LH performed with a tissue fusion device involving diagnostic cystoscopy yields major and minor complication rates similar to safely performed $A H$ operations preserving ureters.
\end{abstract}

Key words: abdominal hysterectomy, laparoscopic hysterectomy, diagnostic cystoscopy, ureter dissection.

\section{Introduction}

Hysterectomy is one of the most commonly performed gynecologic procedures around the world. Around 600,000 women in the United States each year undergo a hysterectomy operation, with benign reasons such as menorrhagia, myoma uteri, pelvic pain and uterine prolapse being the major indications [1]. Hysterectomy is performed by one of the three 
methods available, which are abdominal hysterectomy $(\mathrm{AH})$, vaginal hysterectomy $(\mathrm{VH})$ and laparoscopic hysterectomy (LH) [2]. The hysterectomy operation is traditionally performed through an abdominal or vaginal approach [3]. Abdominal hysterectomy $(\mathrm{AH})$ is the most commonly employed hysterectomy technique in Turkey [4].

In 1989, laparoscopic or laparoscopically assisted vaginal hysterectomy was introduced by Reich et al. as an alternative method to abdominal hysterectomy [5]. Factors such as diminished scar tissue formation, shorter length of hospital stay and early return to daily activities and work compared to AH have attracted interest to laparoscopic hysterectomy [6]. The rate of LH for benign disease was reported to be $11.8 \%$ in an analysis conducted in the United States in 2007 [7]. A survey conducted in Australia reported that many gynecologists had a desire to increase their rate of LH [8]. To date, numerous randomized controlled trials and systematic reviews comparing hysterectomy methods have been conducted [9]; however, there is still no consensus on the optimal hysterectomy method [10].

The rates of intraoperative and postoperative complications have been reported to be increased in conjunction with the increased use of LH [11]. Injury to the great vessels, bowel injury and injury to the urinary tract (bladder or ureter) have become more pronounced with $\mathrm{LH}$ than with traditional $\mathrm{AH}$ [12]. The most striking finding in comparison of abdominal versus $\mathrm{LH}$ methods was that $\mathrm{LH}$ was associated with an approximately 2.6-fold increased risk of urinary tract injury. In particular, LH with the indication of advanced stage endometriosis brings about 1-10\% risk of bowel injury [13, 14].

Higher rate of morbidities occurring after $\mathrm{LH}$ performed for benign indications and which mostly involve the urinary tract could be associated with numerous factors such as the laparoscopic procedure itself being difficult to perform, lack of surgical practice, the patient having a history of previous surgery and the size of the uterus. Primum non nocere, a Latin maxim, translates as "First, do no harm". Keeping this phrase in our guidance, it still remains an unanswered question whether, in the absence of gynecological malignancy and under elective conditions, to perform $\mathrm{AH}$, offering a safer approach, or to perform an $\mathrm{LH}$ procedure where due precautions are taken for possible complications.

\section{Aim}

In this study, we aimed to compare LH operations performed with a tissue fusion device accompanied by intraoperative diagnostic cystoscopy with traditional $\mathrm{AH}$ operations involving bilateral ureteral dissection.

\section{Material and methods}

Eighty-five patients, who underwent an operation in the Department of Obstetrics and Gynecology in Bucak State Hospital and in the Department of Obstetrics and Gynecology in Düzce University Faculty of Medicine between November 2010 and November 2012, were included in this study. Thirty-five patients underwent $\mathrm{AH}$ with or without removal of tubes/ovaries and with concurrent ureteral dissection (UD), whereas 50 patients underwent LH using a tissue fusion device with or without removal of tubes/ovaries and accompanied by diagnostic cystoscopy (CYS). All operations were performed by the same surgeons. Data of the patients were retrieved retrospectively from the records of Bucak State Hospital and Düzce University Faculty of Medicine Training and Research Hospital. Signed informed consent was obtained from all patients undergoing surgery. The study received ethics approval from the Ethics Committee of Non-invasive Research at Düzce University, School of Medicine (Decision number: 2013/389).

In our study, women whose periods have stopped for one complete year were considered menopausal. The history of abdominal surgery was considered remarkable if the patient had previously undergone Caesarean section or any abdominal operation. Past medical history was considered remarkable for systemic disease if the patient had any systemic disease such as previously diagnosed diabetes, hypertension, renal failure or thyroid dysfunction.

All patients were administered preoperative antibiotic prophylaxis with $1 \mathrm{~g}$ of cefazolin. Patients were given a liquid diet for 1 day before the surgery. Bowel preparation was performed by the administration of oral laxatives one day prior to surgery and rectal enemas administered every $8 \mathrm{~h}$. Operation time is defined as the time frame in minutes between the initiation and cessation of the procedure. The difference in hematocrit and hemoglobin levels measured before and at $6 \mathrm{~h}$ after surgery is termed as $\Delta \mathrm{Htc}$ and $\Delta \mathrm{Hb}$. 
Postoperative fever was defined as body temperature above $100.4^{\circ} \mathrm{F}\left(38^{\circ} \mathrm{C}\right)$ in two consecutive measurements taken with 4-hour intervals [15]. Highest measurements of the patients recorded during their hospital stay were included in the study. The patients were considered to have surgical site infection if they met the Centers for Disease Control and Prevention criteria for surgical site infection: i) infection occurs within 30 days after the operation, ii) purulent drainage from the incision, iii) organisms isolated from the culture of fluid obtained from primarily closed operation site, iv) diagnosis of infection by the surgeon, and v) presence of surgical site requiring reopening [16].

The patients were considered to have developed cardiopulmonary complications if pulmonary embolism, cardiac failure or heart and lung problems resembling pulmonary edema had occurred in the postoperative period. Patients were deemed positive for re-operation if they had undergone a second operation. Genitourinary fistula is defined as the development of abnormal communication between the vaginal mucosa and bladder or ureter, which did not exist in the preoperative period and which caused unimpeded leakage of urine. Analgesic requirement in the postoperative period was defined as the total number of tenoxicam $20 \mathrm{mg}$ vials required in the postoperative period. Patients with a uterus measuring 16 weeks gestational size or larger underwent AH + UD. Apart from that, no exclusion criteria were employed for patients undergoing $\mathrm{LH}+\mathrm{CYS}$.

\section{Surgical technique}

General anesthesia or spinal anesthesia was administered in patients who underwent AH + UD. The abdomen was opened by Pfannenstiel incision. Richardson-type hysterectomy was used in all patients. During the procedure, loose retroperitoneal connective tissue was dissected with the index finger or with the tail end of tissue forceps. The external iliac artery coursing along the medial aspect of the psoas muscle was exposed. The bifurcation of the common iliac artery was exposed with blunt dissection. The ureter was immobilized at the point where it crosses the pelvic fossa. The ureter was dissected along its course running parallel to the internal iliac artery and immobilized adjacent to the uterine artery [17]. Before completion of the operation, the integrity of the ureters, ureteral peristalsis and the diameter of the ureters were examined.
All LH + CYS operations were conducted under general anesthesia. For better manipulation of the uterus, a uterine manipulator with a vaginal apparatus was inserted into the uterine cavity; it fits over the vaginal fornices and provides identification of the cervico-vaginal margin during intra-abdominal inspection. The 10-mm laparoscope was inserted through the umbilical trocar that was introduced into the abdominal cavity by a vertical incision. Two $5-\mathrm{mm}$ trocars were placed in the left and right lower quadrants lateral to the rectus abdominis muscle and inferior epigastric vessels [2]. Finally, a 10-mm trocar was placed $3 \mathrm{~cm}$ below the left costal margin in the midclavicular line (Palmer's point). A 10-mm tissue fusion device (Ligasure ${ }^{\circledR}$ ) was inserted through the trocar placed on Palmer's point. A 5-mm dissector and grasping forceps were inserted through the trocars placed in the left and right lower quadrants. A $5-\mathrm{mm}$ tissue fusion device (Ligasure ${ }^{\circledR}$ ) was inserted into the abdominal cavity with 5-mm or 10-mm trocars, when required. The operation was carried out by suspending the uterus from the pelvic floor with the aid of the manipulator while paying attention to the possible route of the ureter just beneath the peritoneum. Round ligaments in both sides were dissected and hemostasis was achieved using a tissue fusion device (Ligasure ${ }^{\circledR}$ ). The infundibulopelvic ligament in patients undergoing salpingo-oophorectomy, otherwise the utero-ovarian ligament, was dissected by a tissue fusion device (Ligasure ${ }^{\circledR}$ ) and hemostasis was achieved. The anterior and posterior sheets of the peritoneum were unfolded to expose the uterine artery and hemostasis was achieved by using a tissue fusion device. The vesico-uterine fold of the peritoneum was dissected and freed from the bladder, uterus and upper portion of the vagina according to the procedure as described by Tanaka et al. [2]. After dissecting the cardinal ligaments, vaginal walls were incised circumferentially using monopolar needle cautery while stretching over the vaginal fornices with the aid of the manipulator. The surgical specimen was removed via the vagina. Uterine morcellation was used to remove the specimen, where necessary. Long-term absorbable sutures were placed intravaginally to close the vaginal cuff and retroperitoneum [2]. Finally, the abdominal cavity was evaluated by laparoscopy for hemostasis. The patients then underwent diagnostic cystoscopy using a rigid cystoscope. A cystoscope with 4-mm outer diameter, $30^{\circ}$ direction of view and $22 \mathrm{Fr}$ outer sheath was 
inserted into the bladder. After the bladder was filled with saline, the interureteric fold and both ureteral openings were determined. The bladder wall was systematically inspected and a jet of urine spurting was noted from both ureteral openings. Pre-renal reasons were considered in the first place when the bilateral jet of urine spurting was found to be absent or minimal, and the patient was accordingly administered a bolus injection of intravenous fluids and/or diuretics. Ureteral catheterization was performed using $6 \mathrm{Fr}$ or $8 \mathrm{Fr}$ ureteral catheters in cases in which the absence of ureteral jet stream persisted or in the case of unilateral absence of a jet of urine spurting.

\section{Statistical analysis}

Mean, standard deviation, proportion and frequency were used in descriptive statistics. The distribution of data was determined by the KolmogorovSmirnov test. Quantitative data were analyzed using an independent samples $t$-test and Mann-Whitney $U$ test. The $\chi^{2}$ test was used in the analysis of qualitative data. The SPSS 20.0 software package was used in statistical analyses. Values of $p<0.05$ were considered statistically significant.

\section{Results}

We compared a total of 85 patients who underwent either $\mathrm{AH}+\mathrm{UD}$ or $\mathrm{LH}+\mathrm{CYS}$. The two groups were not found to be significantly different in terms of age $(p=0.221)$, gravida $(p=1.000)$, parity $(p=$ $0.205)$, proportion of menopausal women $(p=0.765)$, past history of abdominal surgery $(p=0.926)$, or presence of systemic disease $(p=0.313)$ (Table I). The indications for the operations in the two groups are shown in Table II. The most common indication for the operation was myoma uteri in both patients undergoing $\mathrm{AH}+\mathrm{UD}(n=20,57.1 \%)$ and patients undergoing $\mathrm{LH}+\mathrm{CYS}(n=21,42.0 \%)$. Adnexal mass was found to be the second most common indication for the operation in the two groups (Table II).

Among patients who underwent AH + UD, 9 (25.7\%) were administered spinal/epidural anesthesia and 26 (74.3\%) were administered general anesthesia. All patients who underwent $\mathrm{LH}+\mathrm{CYS}$ were administered

Table I. Demographic characteristics of patients

\begin{tabular}{|lccc|}
\hline Variable & AH + UD & LH + CYS & Value of $p^{*}$ \\
\hline Age, mean \pm SD & $50.6 \pm 9.5$ & $48.4 \pm 6.8$ & 0.221 \\
\hline Parity, mean \pm SD & $2.6 \pm 1.2$ & $2.7 \pm 1.1$ & 0.205 \\
\hline Menopause, $n(\%)$ & $22(62.9)$ & $33(66.0)$ & 0.765 \\
\hline Surgical history, $n(\%)$ & $8(22.9)$ & $11(22.0)$ & 0.926 \\
\hline Systemic disease, $n(\%)$ & $7(20.0)$ & $6(12.0)$ & 0.313 \\
\hline
\end{tabular}

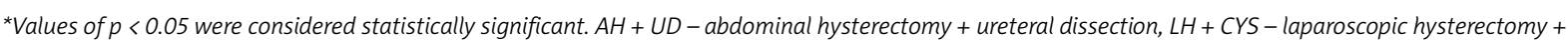
diagnostic cystoscopy

Table II. Indications for the operations

\begin{tabular}{|lcccc|}
\hline Variable & \multicolumn{2}{c}{ AH + UD } & \multicolumn{2}{c|}{ LH + CYS } \\
\cline { 2 - 5 } & $n$ & $\%$ & 10 & $\%$ \\
\hline AUK & 6 & 17.1 & 1 & 20.0 \\
\hline Descensus uteri & 0 & 0.0 & 7 & 14.0 \\
\hline End. hyperpl. & 1 & 2.9 & 21 & 42.0 \\
\hline Myoma & 21 & 60.0 & 11 & 22.0 \\
\hline Adnexal mass & 7 & 20.0 & & $n$ \\
\hline
\end{tabular}

$A H+U D$ - abdominal hysterectomy + ureteral dissection, $L H+C Y S-l$ laparoscopic hysterectomy + diagnostic cystoscopy 
general anesthesia. The operation time was found to be significantly longer in patients who underwent LH + CYS ( $p=0.0001)$. Delta Htc was significantly higher in patients who underwent AH + UD ( $p=0.0001)$. Likewise, $\Delta \mathrm{Hb}$ was significantly higher in patients who underwent $\mathrm{AH}+\mathrm{UD}(p=0.002)$. No significant difference was found between $A H+U D$ and $L H+C Y S$ patient groups in terms of intraoperative complications including ureteral injury, bowel injury and bladder injury ( $p=0.378, p=1.000$ and $p=1.000$, respectively) (Table III).

Patients who underwent $\mathrm{AH}+\mathrm{UD}$ were not significantly different from those who underwent $\mathrm{LH}+$ CYS in terms of mean body temperature or postoperative blood transfusion requirements $(p=0.051$ and $p=0.210$, respectively). Mean parenteral analgesic requirement in the postoperative period was found to be significantly lower in patients who underwent $\mathrm{LH}$ + CYS $(p=0.005)$. In our study, postoperative length of hospital stay was found to be significantly higher in patients who underwent AH + UD ( $p=0.0001)$. No statistically significant difference was found between the two groups in terms of postoperative cardiopulmonary complications, re-operation rate or occurrence of genitourinary fistula $(p=1.000, p=1.000$ and $p=1.000$, respectively). However, the rate of sur- gical site infection in the postoperative period was found to be higher among patients who underwent $\mathrm{AH}+\mathrm{UD}(p=0.030)$ (Table IV).

\section{Discussion}

Decreasing health-care costs and increasing the quality of life are targeted by selecting the optimal hysterectomy method [18]. Laparoscopic hysterectomy appears to be an appropriate alternative method. Technical drawbacks, potential complications and lack of surgical experience may hamper LH coming into common practice [19]. Abdominal hysterectomy is commonly performed in current practice. The rates of $\mathrm{AH}$ have been reported to be $80 \%$ in Denmark, $63 \%$ in the United States and $67.9 \%$ in Turkey [1, 4, 20]. Several methods have been introduced in order to decrease the high rate of major complications which is perhaps the main limitation associated with LH [20]. Laparoscopic hysterectomy brings about a higher rate of lower urinary tract injury compared to $\mathrm{AH}$ [21]. There is, however, a lack of consensus regarding the protective measures against urinary tract injury occurring during LH. In 2007, ACOG recommended the use of cystoscopy in patients with suspicion of injury, whereas in 2012, the American

Table III. Comparison of intraoperative variables

\begin{tabular}{|lccc|}
\hline Variable & AH + UD & LH + CYS & Value of $p^{*}$ \\
\hline Operation time, mean \pm SD [min] & $109.57 \pm 27.07$ & $145.50 \pm 37.75$ & 0.0001 \\
\hline Hematocrit values, mean \pm SD [\%] & & & 0.154 \\
\hline Pre-op & $37.36 \pm 4.28$ & $35.82 \pm 5.21$ & 0.142 \\
\hline Post-op & $31.63 \pm 3.51$ & $32.86 \pm 3.94$ & 0.0001 \\
\hline$\Delta$ Htc & $-5.71 \pm 3.19$ & $-2.86 \pm 4.11$ & 0.286 \\
\hline Hemoglobin values, mean \pm SD [g/dl] & & & 0.131 \\
\hline Pre-op & $15.01 \pm 16.09$ & $11.66 \pm 2.49$ & 0.002 \\
\hline Post-op & $10.49 \pm 1.27$ & $10.96 \pm 1.35$ & 0.378 \\
\hline$\Delta$ Hb & $-4.43 \pm 16.32$ & $-0.64 \pm 2.21$ & 1.000 \\
\hline Ureteral injury, $n(\%)$ & $3(8.6)$ & $2(4.0)$ & 1.000 \\
\hline Bowel injury, $n(\%)$ & $0(0.0)$ & $1(2.0)$ & $1(0.0)$ \\
\hline Bladder injury, $n(\%)$ & $0(0.0)$ & & \\
\hline
\end{tabular}

*Values of $p<0.05$ were considered statistically significant, AH + UD - abdominal hysterectomy + ureteral dissection, LH + CYS - laparoscopic hysterectomy + diagnostic cystoscopy 
Table IV. Comparison of postoperative variables

\begin{tabular}{|lccc|}
\hline Variable & AH + UD & LH + CYS & Value of $p^{*}$ \\
\hline Fever, mean \pm SD $\left[{ }^{\circ} \mathrm{C}\right]$ & $36.95 \pm 0.57$ & $36.74 \pm 0.40$ & 0.051 \\
\hline Blood transfusion, $n(\%)$ & $1(2.9)$ & $4(8.0)$ & 0.210 \\
\hline Number of post-op analgesics, mean \pm SD & $3.26 \pm 3.59$ & $2.08 \pm 1.91$ & 0.005 \\
\hline Post-op complications, $n(\%)$ & & $2(4.0)$ & 1.000 \\
\hline Cardiopulmonary complications & $2(5.7)$ & $1(2.0)$ & 1.000 \\
\hline Re-operation & $0(0.0)$ & $1(2.0)$ & 1.000 \\
\hline Genitourinary fistula & $0(0.0)$ & $1(2.0)$ & 0.030 \\
\hline Surgical site infection & $5(14.3)$ & $3.56 \pm 1.11$ & 0.0001 \\
\hline Length of hospital stay, mean \pm SD & $5.54 \pm 2.28$ & & \\
\hline
\end{tabular}

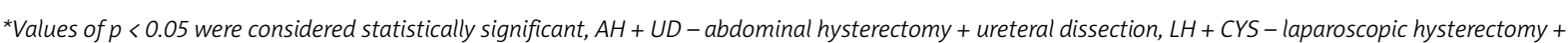
diagnostic cystoscopy

Association of Gynecological Laparoscopy recommended the use of cystoscopy in all patients [22, 23]. Tanaka et al. reported that ureteral catheterization offers a practical and safe method particularly in determining ureteral injuries [2]. Tissue fusion devices do not just ligate vessels but also ligate tissue and luminal structures such as ureters. Cystoscopy helps to detect some injuries that would have otherwise been missed during laparoscopic surgery. The rates of injuries identified are up to 5-fold higher when routine cystoscopy is performed [12]. Ribeiro et al. offered to investigate a possible complication of ureter ligation and cutting when the intraoperative cystoscopy during LH showed no flow through the ureter. They claimed that intra-operative cystoscopy allows for early recognition and treatment of especially obstructive ureteral injuries and may reduce the rate of late postoperative complications during advanced laparoscopic procedures [24]. Hove et al. investigated ureteral injuries in gynecological surgery and reported that 44 of 136 ureteral injuries could potentially have been avoided using established surgical practices, most importantly by exposing the ureter via dissection [25]. In our study, we compared patients who underwent AH + UD with those who underwent $\mathrm{LH}+\mathrm{CYS}$ performed with a tissue fusion device in terms of urinary tract injuries and other intraoperative and postoperative variables.

Past history of pelvic or other surgery, age, and parity determine the type of hysterectomy method
$[26,27]$. The percentage of $\mathrm{AH}$ increased with increasing age, as compared to LH [28]. However, our study was conducted on a homogeneous group of patients and no specific exclusion criteria were set for LH + CYS other than the uterus measuring larger than at 16 weeks of gestation. Demographic characteristics were not significantly different between the two groups in our study.

The most common indication for the operation was myoma uteri for both groups in our study. Myoma uteri was the most common indication for both $\mathrm{AH}$ and $\mathrm{LH}$ in some previous reports, a finding similar to that in our study [4-21]. The second most common indication in our study was an adnexal mass, whereas some of the studies performed to determine ureteral injuries associated with LH reported adenomyosis being the second most common indication for the operation $[2,29]$.

Laparoscopic hysterectomy is a technically more difficult procedure than AH. Similar to our findings ( $p=0.0001$ ), many other studies and reviews have shown that mean operation time was longer in $\mathrm{LH}$ than in $\mathrm{AH}[4,30]$. Although we performed diagnostic cystoscopy in conjunction with $\mathrm{LH}$, mean operation time was $145.50 \pm 37.75 \mathrm{~min}$, which is not different to the reported figures in similar studies [4, 5]. In our study, ureter dissection during $\mathrm{AH}$ was performed in accordance with the surgical techniques described in TeLinde's Operative Gynecology book. The ureters were evaluated for the presence of injury, ureteral 
peristalsis and their diameters. Mean operation time for $\mathrm{AH}+$ UD was found to be $109.57 \pm 27.07 \mathrm{~min}$. Mean operation time for patients undergoing only $\mathrm{AH}$ was $124.4 \pm 64.7 \mathrm{~min}$ [26], and 125 minutes in other studies [4]. Although we performed ureteral dissection during $\mathrm{AH}$, mean operation time in our study was similar to those in large series reported in the literature, in which ureteral dissection was not performed.

The decline in hematocrit $(\Delta \mathrm{Htc})$ and hemoglobin $(\Delta \mathrm{Hb})$ levels was significantly higher in our patients who underwent AH + UD ( $p=0.0001$ and $p=0.002$, respectively). Most studies comparing $\mathrm{AH}$ and $\mathrm{LH}$ reported estimated blood loss to be significantly lower with $\mathrm{LH}[4,9,13]$. According to our results, $\mathrm{LH}+$ CYS could be recommended as the first choice of hysterectomy method in order to reduce morbidity rates in clinically anemic patients or in patients with borderline low $\mathrm{Hb}$ and Htc levels. Further studies are indicated to determine the contribution of ureteral dissection performed in conjunction with $\mathrm{AH}$ to estimated blood loss. We could not find any studies that described the amount of blood loss in $\mathrm{AH}$ concomitant with UD.

In our study, no statistically significant difference was found between the AH + UD and LH + CYS patient groups in terms of intraoperative major complications such as ureteral injury, bowel injury and bladder injury $(p=0.378, p=1.000, p=1.000$, respectively). A comprehensive review previously showed that the rate of major complications was not affected by the type of hysterectomy method employed [30]. Nieboer et al. compared hysterectomy methods and reported higher incidence of urinary tract injuries with LH [9]. Klimczak et al. reported that conversion from laparoscopic hysterectomy to laparotomy and thermal injury to the ureter can occur at the beginning of the learning curve of LH [31]. Garry indicated that studies comparing hysterectomy methods are generally multicenter and far from being objective, and complication rates do not allow clear assessment [10]. In our study, operations were conducted by the same surgeons working in two different centers located in different cities.

Ureterovaginal fistula occurred in one patient, who underwent $\mathrm{LH}+\mathrm{CYS}$ with a tissue fusion device, on postoperative day 10 , although the bladder mucosa appeared intact and a bilateral ureteral jet of urine spurting was observed on intraoperative cystoscopy in this patient. Lim et al. reported that ureter injuries to a certain extent are caused by the electrocautery and surface in the days following the operation [21]. Urologists claim that inspection of the ureters is the most important diagnostic tool in intraoperative diagnosis of ureteral injuries. Inspection offers a $89.3 \%$ diagnostic advantage over pyelography in intraoperative diagnosis of ureteral injury [32]. We believe that ureters must be visualized during $\mathrm{AH}$ and be inspected for their integrity, and presence of hemorrhage and contusion. We performed $\mathrm{AH}$ by exposing ureters along their courses.

Patients who underwent AH + UD were not significantly different from those who underwent $\mathrm{LH}+\mathrm{CYS}$ in terms of postoperative blood transfusion requirements $(p=0.210)$. None of our patients required preoperative blood transfusion. Large series comparing $\mathrm{AH}$ and $\mathrm{LH}$ showed no significant difference between blood transfusion requirements in the two groups $[13,33,34]$. None of our patients developed febrile morbidity in the postoperative period. Patients who underwent $\mathrm{AH}+\mathrm{UD}$ were not significantly different from those who underwent $\mathrm{LH}+\mathrm{CYS}$ in terms of mean postoperative body temperature $(p=0.051)$. Studies by Kluivers et al. and Ribeiro et al. both demonstrated no difference in terms of febrile morbidity between patients who underwent $\mathrm{AH}$ or $\mathrm{LH}[13$, 33]. Our findings on febrile morbidity seem consistent with the literature. The retroperitoneum was opened during $\mathrm{AH}$ and ureters were exposed along their courses in the neighborhood of the operational field in our study. Cystoscopy was performed during LH in our study. Although these procedures in addition to classic $\mathrm{AH}$ and $\mathrm{LH}$ would be anticipated to increase febrile morbidity, none of our patients experienced postoperative fever. The incidence of urinary tract infections has been shown to be higher in gynecological procedures where ureteral catheterization is employed [35]. We consider that employing diagnostic cystoscopy for the sole purpose of inspecting the bladder wall and observing ureteral jet streams might have prevented postoperative febrile morbidity in our study. Moreover, basic principles of abdominal surgery were followed while dissecting the ureters.

No statistically significant difference was found between the two groups in terms of postoperative cardiopulmonary complications, re-operation rate or occurrence of genitourinary fistula ( $p=1.000, p=1.000$ and $p=1.000$, respectively). It was previously reported that pneumoperitoneum could hamper venous circulation in the lower extremity and cause deep 
venous thrombosis or pulmonary embolism [36]. Reoperation at the early postoperative period after hysterectomy is mostly due to hemorrhage. Sutasanasuang performed $60 \mathrm{AH}$ and $\mathrm{LH}$ operations and none of the patients required re-operation [37]. In a large series of patients comparing hysterectomy methods, $0.4 \%$ of the patients required re-operation after $\mathrm{AH}$ whereas none of the patients undergoing $\mathrm{LH}$ required re-operation [4]. Ureteral dissection and diagnostic cystoscopy added to conventional $\mathrm{AH}$ and $\mathrm{LH}$ procedures did not increase the risk of re-operation.

Many studies have demonstrated lower parenteral analgesic requirement in the postoperative period and shorter length of stay in patients who underwent LH $[4,10,36]$. In our study, mean parenteral analgesic requirement in the postoperative period was found to be significantly lower and length of stay was shorter in patients who underwent $\mathrm{LH}+\mathrm{CYS}$ ( $p=0.005, p=0.0001$, respectively). Use of cystoscopy during $\mathrm{LH}$ in an attempt to reduce lower urinary tract injuries did not influence postoperative analgesic requirement or the length of hospital stay. The rate of surgical site infection in the postoperative period was found to be higher among patients who underwent AH + UD $(p=0.030)$. Nieboer et al. reviewed the results of 24 studies and found that surgical site infection is more frequently reported after $\mathrm{AH}$ [9]. Although we performed diagnostic cystoscopy in conjunction with $\mathrm{LH}$, the incidence of surgical site infection was still lower than in $\mathrm{AH}$.

\section{Conclusions}

We conclude that diagnostic cystoscopy followed by LH operations yields major and minor complication rates similar to safely performed $\mathrm{AH}$ operations preserving ureters. It must, however, be kept in mind that ureteral injuries are caused by the electrocautery and perioperative diagnostic cystoscopy may fail to identify such injuries. Comprehensive research on a larger scale is needed in order to investigate safer and easy-to-perform methods in LH that would reduce urinary tract complications and all-cause complication rates. This may, therefore, close the gap between the rates of abdominal hysterectomy and laparoscopic hysterectomy.

\section{References}

1. Farquhar CM, Steiner CA. Hysterectomy rates in the United States 1990-1997. Obstet Gynecol 2002; 99: 229-4.
2. Tanaka Y, Asada H, Kuji N, Yoshimura Y. Ureteral catheter placement for prevention of ureteral injury during laparoscopic hysterectomy. J Obstet Gynaecol Res 2008; 34: 67-72.

3. Clayton RD. Hysterectomy. Best Pract Res Clin Obstet Gynaecol 2006; 20: 73-87.

4. Doğanay M, Yildiz Y, Tonguc E, et al. Abdominal, vaginal and total laparoscopic hysterectomy: perioperative morbidity. Arch Gynecol Obstet 2011; 284: 385-9.

5. Reich H, De Caprio J, McKlynn F. Laparoscopic hysterectomy. J Gynecol Surg 1989; 5: 213-6.

6. Koh LW, Koh PH, Lin LC, et al. A simple procedure for the prevention of ureteral injury in laparoscopic-assisted vaginal hysterectomy. J Am Assoc Gynecol Laparosc 2004; 11: 167-9.

7. Wu JM, Wechter ME, Geller EJ, et al. Hysterectomy rates in the United States, 2003. Obstet Gynecol 2007; 110: 1091-5.

8. Englund M, Robson S. Why has the acceptance of laparoscopic hysterectomy been slow? Results of an anonymous survey of Australian gynecologists. J Minim Invasive Gynecol 2007; 14: 724-8.

9. Nieboer TE, Johnson N, Lethaby A, et al. Surgical approach to hysterectomy for benign gynaecological disease. Cochrane Database Syst Rev 2009; 3: CD003677.

10. Garry R. The future of hysterectomy. BJOG 2005; 112: 133-9.

11. Richardson RE, Bournas N, Magos AL. Is laparoscopic hysterectomy a waste of time? Lancet 1995; 345: 36-41.

12. Gilmour DT, Das S, Flowerdew G. Rates of urinary tract injury from gynecologic surgery and the role of intraoperative cystoscopy. Obstet Gynecol 2006; 107: 1366-72.

13. Kluivers KB, Hendriks JC, Mol BW, et al. Quality of life and surgical outcome after total laparoscopic hysterectomy versus total abdominal hysterectomy for benign disease: a randomized, controlled trial. J Minim Invasive Gynecol 2007; 14: 145-52.

14. Kluivers KB, Johnson NP, Chien P, et al. Comparison of laparoscopic and abdominal hysterectomy in terms of quality of life: a systematic review. Eur J Obstet Gynecol Reprod Biol 2008; 136: 3-8.

15. Lyon DS, Jones JL, Sanchez A. Postoperative febrile morbidity in the benign gynecologic patient. Identification and management. J Reprod Med 2000; 45: 305-9.

16. Horan TC, Gaynes RP, Martone WJ, et al. CDC definitions of nosocomial surgical site infections, 1992: a modification of CDC definitions of surgical wound infections. Am J Infect Control 1992; 20: 271-4.

17. Rock JA, Jones HW. Telinde's Operative Gynecology. $9^{\text {th }}$ edn. Lippincott Williams \& Wilkins, Philadelphia 2003.

18. Bell MC, Torgerson J, Seshadri-Kreaden U, et al. Comparison of outcomes and cost for endometrial cancer staging via traditional laparotomy, standard laparoscopy and robotic techniques. Gynecol Oncol 2008; 111: 407-11.

19. Einarsson JI, Matteson KA, Schulkin J, et al. Minimally invasive hysterectomies-a survey on attitudes and barriers among practicing gynecologists. J Minim Invasive Gynecol 2010; 17: 167-75.

20. Gimbel H, Settnes A, Tabor A. Hysterectomy on benign indication in Denmark 1988-1998. A register based trend analysis. Acta Obstet Gynecol Scand 2001; 80: 267-72. 
21. Lim MC, Lee BY, Lee DO, et al. Lower urinary tract injuries diagnosed after hysterectomy: seven-year experience at a cancer hospital. J Obstet Gynaecol Res 2010; 36: 318-25.

22. ACOG Committee Opinions, 2007.

23. AAGL Advancing Minimally Invasive Gynecology. AAGL Practice Report: Practice guidelines for intraoperative cystoscopy in laparoscopic hysterectomy. J Minim Invasive Gynecol 2012; 19: 407-11.

24. Ribeiro S, Reich H, Rosenberg J, et al. The value of intra-operative cystoscopy at the time of laparoscopic hysterectomy. Hum Reprod 1999; 14: 1727-9.

25. Hove LD, Bock J, Christoffersen JK, Andreasson B. Analysis of 136 ureteral injuries in gynecological and obstetrical surgery from completed insurance claims. Acta Obstet Gynecol Scand 2010; 89: 82-6.

26. David-Montefiore E, Rouzier R, Chapron C, et al. Surgical routes and complications of hysterectomy for benign disorders: a prospective observational study in French university hospitals. Hum Reprod 2007; 22: 260-5.

27. Dorsey JH, Steinberg EP, Holtz PM. Clinical indications for hys terectomy route: patient characteristics or physician preference? Am J Obstet Gynecol 1995; 173: 1452-60.

28. Turner LC, Shepherd JP, Wang L, et al. Hysterectomy surgical trends: a more accurate depiction of the last decade? Am J Obstet Gynecol 2013; 208: 277. e1-7.

29. Ko ML, Lin HW, Chen SC, Pan HS. Should cystoscopy be routinely performed after laparoscopy-assisted vaginal hysterectomy? Minim Invasive Ther Allied Technol 2008; 17: 195-9.

30. Walsh CA, Walsh SR, Tang TY, Slack M. Total abdominal hysterectomy versus total laparoscopic hysterectomy for benign disease: a meta-analysis. Eur J Obstet Gynecol Reprod Biol 2009; 144: 3-7.

31. Klimczak K, Malinowski A, Dejewski C. Choice of route for hys terectomy - evaluation of procedures performed in the gynaecological department of the Tuchola District Hospital, from 2005 to 2008. Videosurgery Miniinv 2010; 5: 14-8.

32. Digiacomo JC, Frankel H, Rotondo MF, et al. Preoperative radiographic staging for ureteral injuries is not warranted in patients undergoing celiotomy for trauma. Am Surg 2001; 67: 969-73.

33. Ribeiro SC, Ribeiro RM, Santos NC, Pinotti JA. A randomized study of total abdominal, vaginal and laparoscopic hysterectomy. Int J Gynaecol Obstet 2003; 83: 37-43.

34. Perino A, Cucinella G, Venezia R, et al. Total laparoscopic hys terectomy versus total abdominal hysterectomy: an assessment of the learning curve in a prospective randomized study. Hum Reprod 1999; 14: 2996-9.

35. Tang KK, Wong CK, Lo SF, Ng TK. Is it necessary to catheterise the bladder routinely before gynaecological laparoscopic surgery? Aust N Z J Obstet Gynaecol 2005; 45: 380-3.

36. Hsieh SW, Lan KM, Luk HN, et al. Massive pulmonary embolism presented as sudden cardiac arrest in the immediate postoperative period after laparoscopic hysterectomy. J Clin Anesth 2003; 15: $545-8$.

37. Sutasanasuang S. Laparoscopic hysterectomy versus total abdominal hysterectomy: a retrospective comparative study. J Med Assoc Thai 2011; 94: 8-16.

Received: 27.04.2013, accepted: 19.10.2013. 